\author{
Redaktion \\ H.G.Bender, Düsseldorf \\ L. Beck, Düsseldorf
}

\section{Abgelegte Kleidungsstücke in der Arztpraxis}

Keine Pflicht zur Verwahrung und Beaufsichtigung

\section{Zum Sachverhalt}

Die Kl. machte Schadensersatzansprüche gegen die beklagte Ärztin wegen des Abhandenkommens eines Pelzcapes im Wert von ca. DM 12 ooo,geltend. Die Kl. behauptete, durch den Hinweis der Praxishelferin auf die im Rezeptionsbereich befindlichen Garderobenhaken habe die Bekl. als Praxisinhaberin eine Verpflichtung zur Verwahrung des Pelzcapes übernommen. Auch sei die Garderobenablage im Empfangsbereich die einzige Möglichkeit zum Ablegen des Pelzcapes gewesen.

Unstreitig befand sich im Untersuchungsraum, in dem sich die Kl. entkleiden mußte, neben der Liege ein Klappstuhl sowie ferner an der Wand ein Haken mit einem Bügel. Klage und Berufung sind ohne Erfolg geblieben.

\section{Aus den Gründen}

Die zulässige Berufung der Kl. bleibt in der Sache ohne Erfolg. Das LG hat die Klage zu Recht abgewiesen. Eine Haftung der Bekl. kommt unter keinem denkbaren rechtlichen Gesichtspunkt in Betracht.

Ein ausdrücklicher Verwahrungsvertrag gemäß $₫ 688$ BGB ist zwischen den Parteien nicht geschlossen worden.
Eine Pflicht zur Verwahrung und Beaufsichtigung ergibt sich auch nicht aus dem mit der Bekl. geschlossenen ärztlichen Behandlungsvertrag. Es entspricht - soweit ersichtlich - einhelliger Meinung in Literatur und Rechtsprechung, daß im Rahmen eines solchen Behandlungsvertrages mit einem Arzt dieser den Patienten allenfalls eine Gelegenheit zum Ablegen von Kleidungsstücken für die Dauer der Behandlung anbietet, daß sich hieraus jedoch nicht zwangsläufig eine Haftungsübernahme bzw. eine entsprechende Verwahrungspflicht des Arztes und seines Personals mit entsprechenden Haftungsfolgen bei Verletzung einer dahingehenden Pflicht ergibt.

Vorliegend sind keine Anhaltspunkte ersichtlich, die die Annahme rechtfertigen könnten, die Bekl. habe entgegen den vorgenannten Grundsätzen der Kl. gegenüber eine besondere Aufbewahrungspflicht übernehmen wollen, noch dafür, daß die Kl. als Patientin nach den Umständen des konkreten Falles davon hätte ausgehen dürfen, die Bekl. übernehme die Verantwortung für eine sichere Verwahrung der von der Kl. abgelegten Sachen.

Das Gericht bestätigt mit diesem Urteil die in Rechtsprechung und Literatur überwiegende Auffassung, daß im
Regelfall Ärzte genau wie Rechtsanwälte oder Gastwirte keine Verwahrungspflicht übernehmen, sondern nur Gelegenheit zum Abstellen von Sachen, insbesondere zum Ablegen von Überkleidung, bieten.

\section{Fazit}

Bietet der Arzt im Rahmen des ärztlichen Behandlungsvertrages den Patienten die Gelegenheit zum Ablegen von Kleidungsstücken für die Dauer der Behandlung, so ergibt sich hieraus noch nicht zwangsläufig eine Verwahrungspflicht und Haftungsübernahme des Arztes und seines Personals. (Leitsatz der Bearbeiterin) OLG Köln, Urt.v. 1.10.1997 - 5 U 63/97 (LG Köln)

Quelle: R. Kiesecker (1999), Keine Pflicht zur Verwahrung und Beaufsichtigung abgelegter Kleidungsstücke durch die Arztpraxis Medizinrecht, Heft 3/99, S. 143-144

Dr.iur. Regine Kiesecker, Stellvertretende Geschäftsführerin der Bezirksärztekammer Südwürttemberg, Haldenhaustraße 11, D-72770 Reutlingen 